\title{
Interaction Of Myxomatosis And Rabbit Haemorrhagic Disease In Wild Rabbit
}

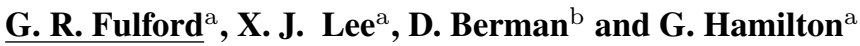 \\ ${ }^{a}$ Faculty of Science and Tech., Queensland University of Technology, 2 George St, Brisbane, QLD, 4001 \\ ${ }^{\mathrm{b}}$ Robert Wicks Pest Animal Research Centre, 203 Tor Street, Toowoomba, QLD 4350 \\ Email: g.fulford@qut.edu.au
}

\begin{abstract}
Increasing resistance of rabbits to myxomatosis in Australia has led to the exploration of Rabbit Haemorrhagic Disease, also called Rabbit Calicivirus Disease (RCD) as a possible control agent. While the initial spread of RCD in Australia resulted in widespread rabbit mortality in affected areas, the possible population dynamic effects of RCD and myxomatosis operating within the same system have not been properly explored. Here we present early mathematical modelling examining the interaction between the two diseases. In this study we use a deterministic compartment model, based on the classical SIR model in infectious disease modelling. We consider, here, only a single strain of myxomatosis and RCD and neglect latent periods. We also include logistic population growth, with the inclusion of seasonal birth rates. We assume there is no cross-immunity due to either disease. The mathematical model allows for the possibility of both diseases to be simultaneously present in an individual, although results are also presented for the case where co-infection is not possible, since co-infection is thought to be rare and questions exist as to whether it can occur. The simulation results of this investigation show that it is a crucial issue and should be part of future field studies.
\end{abstract}

A single simultaneous outbreak of RCD and myxomatosis was simulated, while ignoring natural births and deaths, appropriate for a short timescale of 20 days. Simultaneous outbreaks may be more common in Queensland. For the case where co-infection is not possible we find that the simultaneous presence of myxomatosis in the population suppresses the prevalence of RCD, compared to an outbreak of RCD with no outbreak of myxomatosis, and thus leads to a less effective control of the population. The reason for this is that infection with myxomatosis removes potentially susceptible rabbits from the possibility of infection with RCD (like a vaccination effect). We found that the reduction in the maximum prevalence of RCD was approximately $30 \%$ for an initial prevalence of $20 \%$ of myxomatosis, for the case where there was no simultaneous outbreak of myxomatosis, but the peak prevalence was only $15 \%$ when there was a simultaneous outbreak of myxomatosis. However, this maximum reduction will depend on other parameter values chosen. When co-infection is allowed then this suppression effect does occur but to a lesser degree. This is because the rabbits infected with both diseases reduces the prevalence of myxomatosis.

We also simulated multiple outbreaks over a longer timescale of 10 years, including natural population growth rates, with seasonal birth rates and density dependent (logistic) death rates. This shows how both diseases interact with each other and with population growth. Here we obtain sustained outbreaks occurring approximately every two years for the case of a simultaneous outbreak of both diseases but without simultaneous co-infection, with the prevalence varying from 0.1 to 0.5 . Without myxomatosis present then the simulation predicts RCD dies out quickly without further introduction from elsewhere. With the possibility of simultaneous co-infection of rabbits, sustained outbreaks are possible but then the outbreaks are less severe and more frequent (approximately yearly).

While further model development is needed, our work to date suggests that: 1) the diseases are likely to interact via their impacts on rabbit abundance levels, and 2) introduction of RCD can suppress myxomatosis prevalence. We recommend that further modelling in conjunction with field studies be carried out to further investigate how these two diseases interact in the population.

Keywords: rabbits, calicivirus, myxomatosis, RCD, RHDV, pest control 


\section{INTRODUCTION}

Because of their notorious breeding, adaptability and invasive capabilities, the European rabbit (Oryctolagus cuniculus) has been estimated to cause around $\$ 600$ million damage annually, including the cost of control measures, as well as the endangerment and extinction of native flora and fauna. The Australian government has taken various control methods in attempts to reduce, or at least limit, the rabbit population in Australia. Shooting was one of the more common approaches tried but to little success. Poisoning and warren ripping were also standard conventional control methods but were both labour intensive and expensive. This lead researchers to the consideration of species-selective biological control agents, more specifically, the Myxoma virus in 1950, and later, calicivirus to combat the declining effect of the Myxoma virus.

The Myxoma virus was extremely lethal when first introduced, eliminating in the excess of $99 \%$ of rabbit populations, although the effects varied from site to site. During subsequent years, the virulence of the Myxoma virus experienced a steady decline. The less virulent strain of Myxoma virus is competitively dominant, which explain why although myxomatosis was introduced using the strain with highest virulence (grade I), within a few years, grade III and grade IV were the most abundant strains in the field (Dwyer et al. [1990]). In order to combat the decline, new vectors of the disease, in particular the European and Spanish fleas, were introduced as well as reintroduction of the Myxoma virus in attempts to kick-start the disease again. While these actions increased the impact of the disease temporarily, myxomatosis was still unable to reach the levels of rabbit mortality seen when it was first introduced. Models that study the different strains of myxomatosis in rabbit populations have been developed by Anderson and May [1982].

In 1995, the calicivirus, or rabbit haemorrhagic disease virus (RHDV), was accidentally released in Australia when it was being under consideration as a new potential biological control agent, following the devastating impact of its release in Europe. Calicivirus is the causative agent of rabbit calicivirus disease (RCD) which has a mortality rate of 90-95\% in rabbits that are 3 months or older (Henning et al. [2005]). However, the effects of RCD varied among the sites that were under observation for RCD outbreaks. In particular, it appeared that environmental conditions such as increased rainfall and high temperatures decreased the effectiveness of RCD as a control (Story et al. [2004]). Also reported in Story et al. [2004], there were no occurrence of RCD outbreaks in rabbit populations where the density of susceptibles were less than 12 rabbits $\mathrm{km}^{-2}$ and that the overall effect of RCD was least in sites that were most suitable for rabbits.

It was reported (Mutze et al. [2002]) that at sites that had myxomatosis, the introduction of RCD delayed the yearly outbreaks of myxomatosis. Mutze et al. [2002] suggested that RCD outcompetes myxomatosis when RCD is most active (in spring) due to the longer incubation period of myxomatosis ( $8-10$ days as opposed to 2 days for RCD). Thus, RCD would have killed most rabbits before they become infectious for myxomatosis. However, in certain habitat types, more specifically, swampy areas that may harbour mosquitoes, the shift in the outbreaks of myxomatosis was not observed. It was suggested that it was because mosquitoes were the main vector for myxomatosis and thus it was more favourable over RCD in such areas. Also, it was postulated that for areas with low rabbit density (where RCD would be less effective), myxomatosis could prove to be a better control agent (Story et al. [2004]). Marchandeau et al. [2004] showed statistically that for most of their study areas, prior infection of myxomatosis was a significant factor in the prediction of contraction of RCD, and vice-versa. However, in general, there has been a lack of theoretical research on the combination effect of both myxomatosis and RCD on rabbit populations.

Barlow and Kean [1998] and Barlow et al. [2002] developed models for RCD and suggested that indirect transmission of the virus and juvenile immunity may be required in the model for the disease to persist locally. Then 2-yearly sustained outbreaks were observed. This paper attempts to extend the Barlow and Kean [1998]) model to that incorporates both RCD and myxomatosis and investigate whether myxomatosis can suppress tRCD prevalence. The effect of a seasonal birth-rate is also investigated, but we neglect the effects of juvenile immunity and indirect transmission, to determine if the presence of myxomatosis can also stimulate 2-yearly cycles. 


\section{MODEL DESCRIPTION}

Both the effects of RCD and of myxomatosis on rabbit populations were combined into a single model to investigate possible interactions between the two diseases and the combined effects of the diseases on the rabbit population density. Additional variables are included to incorporate the interaction of the diseases. For this particular model, $S$ refers to the population density that is susceptible to both RCD and myxomatosis, whereas $S_{c}$ and $S_{m}$ refer to the population density susceptible to either RCD but not myxomatosis, or myxomatosis but not RCD respectively. The infectious population is divided into four separate categories: those infectious with RCD, but are not immune to myxomatosis $\left(I_{c}\right)$; those infectious with myxomatosis but are not immune to $\operatorname{RCD}\left(I_{m}\right)$; those infectious with RCD but are immune to myxomatosis $\left(J_{c}\right)$; and those infectious with myxomatosis but have already developed immunity to RCD $\left(J_{m}\right)$.

The recovered population density, $R$, refers to the rabbit population that have survived both infections (RCD and myxomatosis), and hence developed immunity to both the diseases. Immunity obtained by rabbits is assumed to be lifelong for the timescales of interest in this study. For simplicity it is assumed that getting infected with one disease does impact on the disease characteristics of the other disease, such as transmission rate, mortality rate and recovery rate. This assumption is supported by the fact that RCD and myxomatosis are two completely separate diseases so it is unlikely that one will confer any immunity for the other. A compartment diagram for the disease transmission model with both diseases is provided below. In this diagram $\lambda_{c}$ and $\lambda_{m}$ are the force of infection for RCD and myxomatosis, respectively, where

$$
\lambda_{c}=\beta_{c}\left(I_{c}+J_{c}\right), \quad \lambda_{m}=\beta_{m}\left(I_{m}+J_{m}\right), \quad \lambda_{b}=\beta_{c} I_{b}
$$

Here $\beta_{i}$, where $i=m, c$, are transmission coefficients where we have assumed density-dependent transmission in both cases. We have taken the coefficient for rabbits infected with both diseases simultaneously to be the same as for RCD, which is the more virulent, although it is likely the value will be smaller due to the probable diminished contact rates of already sick rabbits. The recovery rate coefficients are $\gamma_{c}$ and $\gamma_{m}$ respectively, for each infectious agent. In this model the possibility for co-infection with both diseases is included, with numbers given by the variable $I_{b}$. It is not currently known if co-infection (simultaneous infection with both diseases) actually occurs in practice. Cooke [2011] suggests that it is rare or nonexistent. RCD infects for 4 days or so, myxomatosis for 15 days. Whether this actually happens or not will also depend in part on seasonal effects. It seems that in SA, the two diseases are unlikely to be active at the same time but it may be true in Queensland that they are. Nevertheless, we include co-infection in the model and later compare the situations where it is possible or not possible. Here we assume that if simultaneous co-infection occurs that the mortality rate is the same as for RCD although in practice it is likely to be higher due to the compromised heath of the rabbit already infected with myxomatosis.

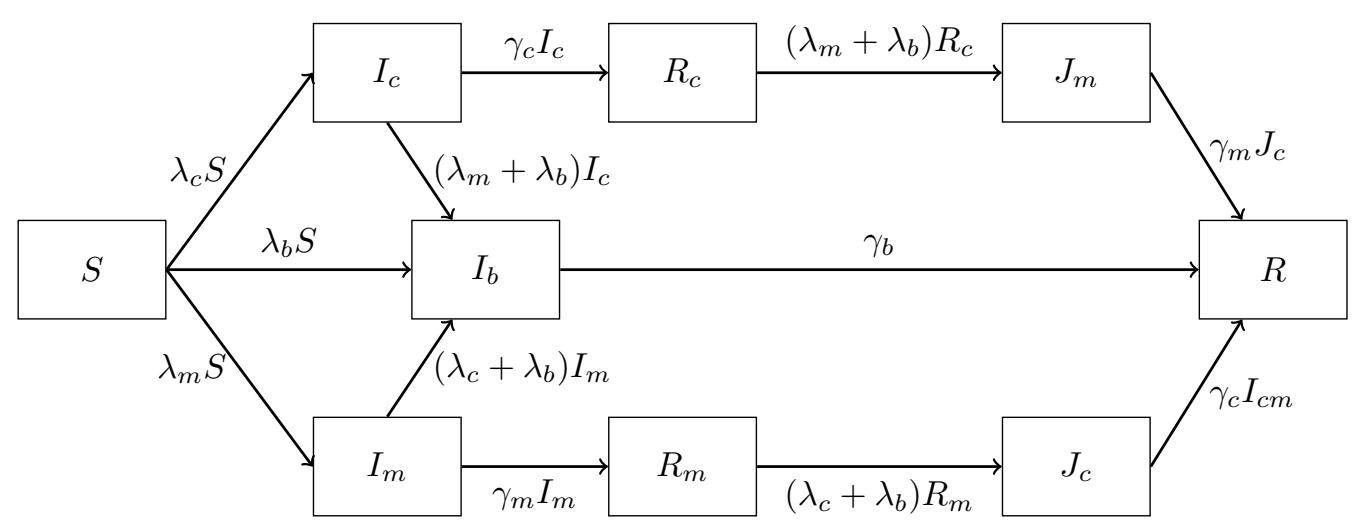

Figure 1. Compartment diagram illustrating the flows between compartments in the model, excluding the natural births and deaths 
Including also the population dynamics, the differential equations corresponding to the compartment diagram in Figure 1 are

$$
\begin{aligned}
\frac{d S}{d t} & =a N-(b+c N) S-\lambda_{c} S-\lambda_{m} S-\lambda_{b} S, \\
\frac{d I_{c}}{d t} & =-(b+c N) I_{c}+\lambda_{c} S-\left(\gamma_{c}+\alpha_{c}\right) I_{c}, \\
\frac{d I_{m}}{d t} & =-(b+c N) I_{m}+\lambda_{m} S-\left(\gamma_{m}+\alpha_{m}\right) I_{m}, \\
\frac{d R_{c}}{d t} & =-(b+c N) R_{m}+\gamma_{c} I_{c}-\left(\lambda_{m}+\lambda_{b}\right) R_{c}, \\
\frac{d R_{m}}{d t} & =-(b+c N) R_{m}+\gamma_{m} I_{m}-\left(\lambda_{c}+\lambda_{b}\right) R_{c}, \\
\frac{d J_{m}}{d t} & \left.=-(b+c N) J_{m}+\lambda_{m}+\lambda_{b}\right) R_{c}-\left(\gamma_{m}+\alpha_{m}\right) J_{m}, \\
\frac{d J_{c}}{d t} & =-(b+c N) J_{c}+\left(\lambda_{c}+\beta_{b}\right) R_{m}-\left(\gamma_{c}+\alpha_{c}\right) J_{c}, \\
\frac{d I_{b}}{d t} & =-(b+c N) I_{b}+\lambda_{b} S+\left(\lambda_{c}+\lambda_{b}\right) I_{m}+\left(\lambda_{m}+\lambda_{b}\right) I_{c}-\left(\alpha_{b}+\gamma_{b}\right)_{b}, \\
\frac{d R}{d t} & =-(b+c N) R+\left(\gamma_{c}+\alpha_{c}\right) J_{c}+\gamma_{m}+\left(\alpha_{m}\right) J_{m}+\left(\gamma_{b}+\alpha_{b}\right) I_{b} .
\end{aligned}
$$

where $N$ is the sum of the nine subpopulations. Here we have assumed constant per-capita birth rate $a$ and a logistic density-dependent death rate $b+c N$. Here $c=(a-b) / K$ where $K$ is the carrying capacity. and $\alpha_{i}$, with $i=m, c$ are the disease-induced death rates. This system of differential equations is solved in MATLAB using the ode 45 routine, which is a robust adaptive time-stepping procedure based on a 4th order Runge-Kutta approach with 5th order error estimation. The options MaxStep (set at 0.1 days) and NonNegative were used for the simulations involving outbreaks over 10 year to prevent the populations from becoming negative and to prevent too small steps being taken so that outbreaks were missed. We found that setting the maximum steps size to 0.05 days gave outbreaks at the same time.

The population dynamics rates (sourced from Barlow and Kean [1998]) were obtained from rabbit populations estimated directly from data. The carrying capacity $K=15$ was provided from Barlow and Kean [1998], based on South Australian data. These are summarised in Table 1. RCD parameters were obtained from Barlow and Kean [1998]. For the models developed in Barlow and Kean [1998], it is mentioned that the addition of an incubation class (i.e. the exposed, $E$, in the network model) did not affect the final outcome of the model, so long as the total duration of the disease (from infection) remained constant and the contact rate increases proportional to the decrease in infectious period. For the rabbits simultaneously infected with both diseases we assume the transmission coefficient $\beta_{b}$ and the diseaseinduced mortality rate coefficient $\alpha_{b}$ to be the same as for RCD but we assume there is no recovery in this case $\left(\gamma_{b}=0\right)$. Myxomatosis in rabbits have been well-studied and can be categorised into different strains by the duration of infection as well as the mortality rate caused by the strain. For the purpose of the investigation to be carried out here, the naturally dominant, and most abundant strain of the myxoma virus in Australia (Grade IIIB) was the only one considered (Anderson and May [1982]). The parameter values were sourced from Aparcio et al. [2004] (for $\beta_{m}$ ) and Anderson and May [1982] (for $\alpha_{m}$ and $\gamma_{m}$ ). An alternate value for $\beta_{m}=0.008$ is given in Dwyer et al. [1990].

\section{RESUlts OF Simulations}

Simulations of the model were produced where the birth and death rates were set to zero to capture the sole effect of the diseases interacting, independent of population density effects. This is representative of a single pair of outbreaks of both diseases passing through the population in a short time scale, with the population having experienced previous outbreaks so that the are some rabbits in the population who have previous recovered from with or both diseases. For the simulations generated in this section, it is assumed that the initial population density $K(K=15)$ comprises $10 \%$ who have recovered from both diseases $(R), 0.1 \%$ infectious with RCD and myxomatosis each, having not caught any other diseases before $\left(I_{c}\right.$ 
Table 1. Disease parameter values, from Barlow and Kean [1998] for RCD and and Aparcio et al. [2004] for myxomatosis.

\begin{tabular}{|c|c|c|}
\hline "Parameter (units) & Symbol & Value \\
\hline $\begin{array}{l}\text { Population } \\
\text { density-independent per-capita birth rate }\left(\text { days }^{-1}\right) \\
\text { density-independent per-capita death rate }\left(\text { days }^{-1}\right) \\
\text { carrying capacity }\left(\text { rabbits ha }^{-1}\right)\end{array}$ & $\begin{array}{c}a \\
b \\
K\end{array}$ & $\begin{array}{c}0.00822 \\
0.00460 \\
15\end{array}$ \\
\hline $\begin{array}{l}\text { RCD } \\
\text { transmission coefficient ( } \text { rabbits }^{-1} \text { ha days }^{-1} \text { ) } \\
\text { per-capita mortality rate due to disease }\left(\text { days }^{-1} \text { ) }\right. \\
\text { per-capita recovery rate }\left(\text { days }^{-1} \text { ) }\right.\end{array}$ & $\begin{array}{l}\beta_{c} \\
\alpha_{c} \\
\gamma_{c}\end{array}$ & $\begin{array}{c}0.173 \\
0.6 \\
0.022\end{array}$ \\
\hline $\begin{array}{l}\text { myxomatosis } \\
\text { transmission coefficient ( } \text { rabbits }^{-1} \text { ha days }{ }^{-1} \text { ) } \\
\text { per-capita mortality rate due to disease }\left(\text { days }^{-1}\right) \\
\text { per-capita recovery rate }\left(\text { days }^{-1}\right)\end{array}$ & $\begin{array}{l}\beta_{m} \\
\alpha_{m} \\
\gamma_{m}\end{array}$ & $\begin{array}{c}0.0053 \\
0.039 \\
0.0098\end{array}$ \\
\hline
\end{tabular}

and $I_{m}$ ), and the rest of the population evenly divided into $S, R_{c}$ and $R_{m}$. An initial prevalence of $10 \%$ for RCD was used in all cases, so the initial numbers for all compartments are

$$
\left(S, I_{c}, I_{m}, R_{c}, R_{m}, J_{m}, J_{c}, I_{b}, R\right)=(0.2,0.1,0.2,0.2,0.2,0,0,0,0.1) K
$$

In Figure 2 the total populations, scaled with respect to the carrying capacity, were plotted along with the prevalence for RCD and for myxomatosis. A comparison is made of where the initial myxomatosis prevalence of $20 \%$ and with no myxomatosis was present initially in the population, and finally with $20 \%$ initial myxomatosis prevalence where simultaneous co-infection is possible. For the scenario where no myxomatosis is present initially, $I_{m}(0)=0$, the initial number of rabbits susceptible to both diseases $S(0)$ is then changed from $0.2 K$ to $0.4 K$.

The case where no co-infection can occur is discussed first. In comparison to a single RCD outbreak, with no myxomatosis present initially, the RCD outbreak with myxomatosis present is suppressed; it reaches a peak RCD prevalence of only slightly over $15 \%$ compared with over $30 \%$. If simultaneous co-infection is allowed then the peak RCD prevalence is still suppressed, but now reaches a value of approximately 0.24. An explanation for the suppression effect, for the case of no co-infection, is that rabbits currently infected with myxomatosis remove possible susceptible rabbits from being infected by RCD; a kind of 'vaccination' effect of myxomatosis on RCD. The total population at 20 days is reduced to 0.46 of the initial population compared to less than 0.3 of the initial population for when there is no current outbreak of myxomatosis. When the possibility of co-infection exists the results are intermediate between the nomyxomatosis and with myxomatosis but no co-infection cases. A possible explanation is the presence of rabbits simultaneously infected with both diseases reduces the prevalence of myxomatosis.

In Figure 3 population dynamics with a seasonal birth rate was assumed, with logistic, density-dependent deaths. The seasonal birth rate, the same as used by Barlow and Kean [1998], has the functional form

$$
f(t)=a\left(1+d \sin \left(\frac{2 \pi t}{365}\right)\right), \quad d=0.8,
$$

to replace the constant rate $a$. Thus, the per-capita birth rate oscillates from $0.2 a$ to $1.8 a$ every year. Using this value results in monthly exponential net growth rates ranging from 0.12 to -0.04 , which is of the same order of magnitude as the values reported in Mutze et al. [2002] for several different field studies in South Australia. For these simulations maximum step-size was set to 0.1 days, to prevent outbreaks being missed, and an option to prevent populations becoming negative was imposed. This gave consistent results with a maximum step size of 0.05 .

For the case where there is no myxomatosis present initially (not shown) then RCD dies out quickly. For the case where both diseases were present initially, with no simultaneous confection allowed, then major outbreaks are sustained, occurring approximately 2 years apart, but irregularly spaced, and with 

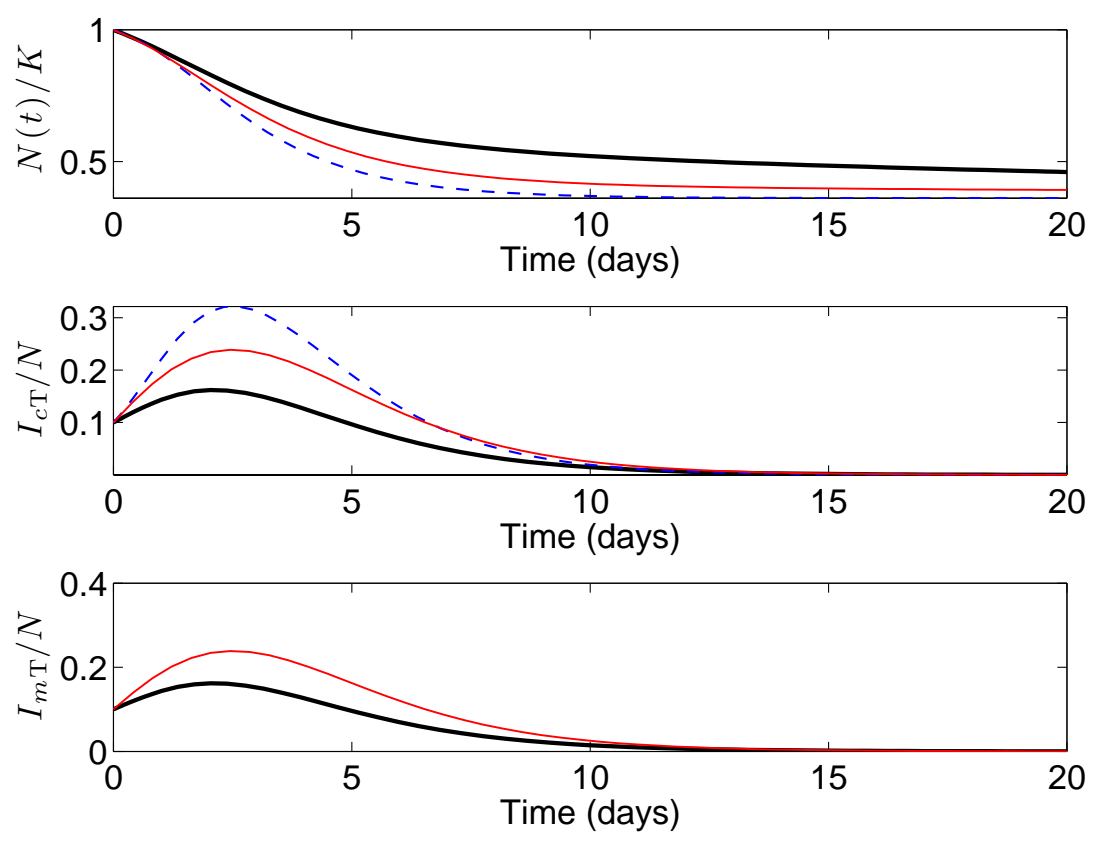

Figure 2. Simulation of model without inclusion of natural birth and deaths over a timescale of a single outbreak. The solid lines are for both diseases present with both RCD and myxomatosis present (black lines for no co-infection and thin red lines for co-infection) and the dashed lines are for RCD alone with no initial myxomatosis. Here $I_{c \mathrm{~T}}$ refers to the total prevalence of RCD, $I_{c \mathrm{~T}}=\left(I_{c}+J_{c}+I_{b}\right) / N$ terms with a similar term $I_{m \mathrm{~T}}$ for the total myxomatosis prevalence.
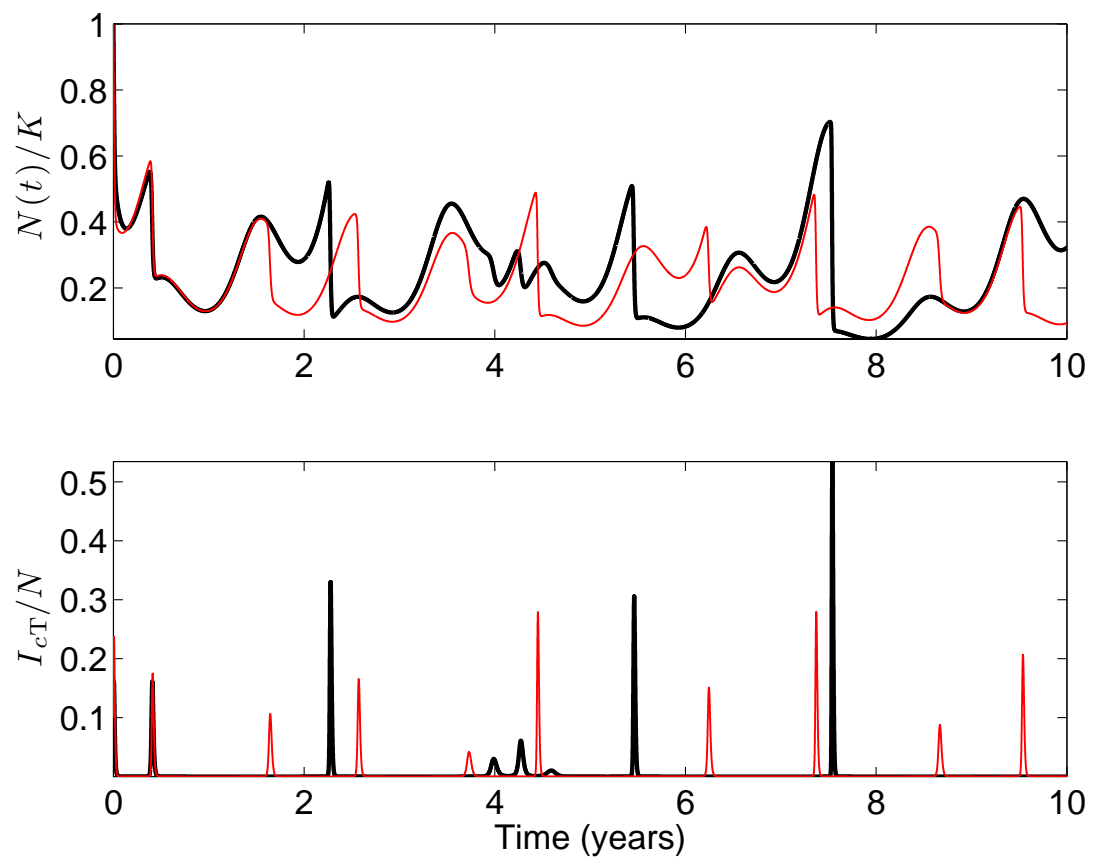

Figure 3. Simulations incorporating both diseases with a density-dependent death rate and a seasonal birth rate. The thick black line is for no simultaneous co-infection and the thin red line allows co-infection to occur. 
peak prevalence of approximately 20-50\% with the exception of one smaller outbreak. The outbreaks of RCD suppress the rabbit populations initially but the populations then recover to approximately $50 \%$ of the carrying capacity before the next outbreak occurs. For the case where simultaneous co-infection can occur the outbreaks are more frequent (approximately 1 per year).

\section{Conclusions}

We have investigated, using a mathematical model in the form of a system of differential equations, the effect of both myxomatosis and RCD on rabbit populations. Our simulations over 20 days have shown that the presence of a concurrent outbreak of myxomatosis can suppress the maximum number of rabbits infected with RCD. This effect occurs both when simultaneous co-infection is not possible as well as when it is possible, but the effect is more pronounced when simultaneous co-infection is not possible. We have also run the simulation over a longer time scale of 10 years including seasonal birth rates and logistic density dependent death rates. The presence of myxomatosis here allows RCD outbreaks to occur, whereas without myxomatosis then RCD dies out. However, this conclusion needs further investigation to determine how robust is this conclusion with respect to changes in parameter values and initial numbers.

It would also be valuable to investigate the effect the different infections have on transmission contact rates, using proximity sensors, such as in Marsh et al. [2010]. Further work is planned to investigate multi-patch models where rabbits can migrate between different habitat patches. These improved models will allow the study of an invasion of RCD infected rabbits into a region populated only by rabbits infected with myxomatosis, and visa versa. Other effects that could be valuable to include are the effects of waning immunity in myxomatosis for looking at longer timescales. This might be valuable in deciding where and when it could be most optimal to introduce RCD to a region as an more effective means of controlling rabbit populations.

\section{REFERENCES}

Anderson, R. and R. May (1982). Coevolution of hosts and parasites. Parasitology 85, 411-426.

Aparcio, J. P., H. G. Solari, and N. Bonino (2004). Competition and coexistence in host-parasite systems: the myxomatosis case. Population Ecology 46, 71-85.

Barlow, N. D., M. Barron, and J. Parkes (2002). Rabbit haemorrhagic disease in New Zealand: field test of a disease-host model. Wildlife Research 29, 649-651.

Barlow, N. D. and J. M. Kean (1998). Simple models for the impact of rabbit calicivirus disease (RCD) on Australasian rabbits. Ecolological Modelling 109, 225-241.

Cooke, B. (2011). Personal communication.

Dwyer, G., S. A. Levin, and L. Buttel (1990). A simulation model of the population dynamics and evolution of Myxomatosis. Ecological Monographs 60, 423-447.

Henning, J., J. Meers, P. Davies, and R. Morris (2005). Survival of rabbit haemorrhagic disease virus (RHDV) in the environment. Epidemiology and Infection 133, 719-730.

Marchandeau, S., S. Bertagnoli, B. Peralta, C. Boucraut-Baralon, J. Letty, and F. Reitz (2004). Possible interaction between myxomatosis and calicivirosis related to rabbit haemorrhagic disease affecting the European rabbit. Veterinary Record 155, 589-592.

Marsh, M., M. Hutchings, S. McLeod, and P. White (2010). Spatial and temporal heterogeneities in the contact behaviour of rabbits. Behavioral Ecology and Sociobiology 65, 183-195.

Mutze, G., P. Bird, J. Kovaliski, D. Peacock, S. Jennings, and B. Cooke (2002). Emerging epidemiological patterns in rabbit haemorrhagic disease, its interaction with myxomatosis, and their effects on rabbit populations in South Australia. Wildlife Research 29, 577-590.

Story, G., D. Berman, R. Palmer, and J. Scanlan (2004). The impact of rabbit haemorrhagic disease on wild rabbit (Oryctolagus cuniculus) population in Queensland. Wildlife Research 31, 183-193. 